\title{
Radial forearm free flap in a patient with an unusual radial artery variation: a case report
}

\author{
Giulio Menichini, Sara Calabrese, Nicola Alfonsi, Marco Innocenti \\ Department of Plastic and Reconstructive Microsurgery, Careggi University Hospital, Florence, Italy
}

\begin{abstract}
Head and neck reconstruction poses unique challenges in rehabilitating surgical defects in terms of integrity, function, and form. The radial forearm free flap (RFFF) has been widely used for defect coverage, especially in the head and neck area, but its versatility allows it to be used for soft-tissue reconstruction in various parts of the body. The vascular features of the flap are quite constant and reliable. Nevertheless, abnormalities of the forearm vascular tree have been described over the decades. We report a case of intraoral reconstruction after verrucous carcinoma recurrence in a 74-year-old woman with an unusual forearm flap, which we called the median forearm free flap, based on a median branch of the radial artery that was preoperatively detected using handheld Doppler ultrasonography. The distally located skin paddle was predominantly supplied by the aberrant median vessel with its perforators. The flap was thus safely harvested with this atypical pedicle. Successful reconstruction of the intraoral defect was achieved, with an uneventful postoperative course.
\end{abstract}

Keywords Perforator flap / Free tissue flaps / Radial artery / Forearm / Head and neck neoplasm
Correspondence: Sara Calabrese Department Plastic and

Reconstructive Microsurgery, Careggi

University Hospital, Via Taddeo Alderotti, 56 Florence 50139, Italy

Tel: $+39-3882579330$

Fax: +39-557948178

E-mail:

calabresesaramed@gmail.com

Received: February 28, 2021 • Revised: July 22, 2021 • Accepted: August 6, 2021

pISSN: 2234-6163 • elSSN: 2234-6171 • https://doi.org/10.5999/aps.2021.00486 • Arch Plast Surg 2021;48:646-650

\section{INTRODUCTION}

Since its introduction [1], the radial forearm free flap (RFFF) has become a workhorse in reconstructive microsurgery due to its thin, pliable, glabrous skin, reliable long vascular pedicle, and the possibility for complex flap designs [2-4]. Although the vascular supply to the volar surface of the forearm is thought to be consistent across individuals, various aberrant patterns have been reported in both the anatomical and surgical literature. Variations such a high origin of the radial artery (RA) from the brachial artery (BA) [5], the presence of a superficial dorsal antebrachial artery, a distal take-off of the RA deep to the pronator teres muscle [6], bifurcation of the RA into superficial and deep branches $[7,8]$, and duplication of the RA [9] have been de- scribed in the English-language literature. Moreover, a few papers have described a persistent median artery in the forearm. In 1995, Mordick [10] reported a case of RFFF that was successfully harvested with an unnamed median vessel arising directly from the axillary artery and paralleling the BA. In a study by Acarturk et al. [11], a median branch arising from the RA was described, together with other coexisting arterial anomalies of the RA system. We present a case wherein a preoperatively detected anomaly of the forearm vascular pattern was encountered while planning a right RFFF for intraoral reconstruction after the resection of a recurrent verrucous carcinoma at the labial commissure, cheek mucosa, and vestibular fornix. Handheld Doppler ultrasonography highlighted the presence of a median branch arising from the RA, which was not associated with oth- 
er abnormalities of the forearm vascular pattern. To the authors' knowledge, this is the first report of a forearm free flap based on a median artery solely supplying the skin of the distal forearm. Successful reconstruction was achieved, with excellent functional and aesthetic outcomes.

\section{CASE}

A 74-year-old woman with no history of smoking or alcohol abuse developed a verrucous carcinoma of the tongue and right tonsillar pillar in 2015. She underwent radical excision of the lesion and unilateral neck dissection (I-IV), followed by reconstruction with a traditional left RFFF. There were no perioperative complications, the donor-site morbidity was minimal, and no forearm arterial anomalies (e.g., median vessels) were found that would prompt suspicion of contralateral abnormalities. Adjuvant radiation therapy was completed in January 2018. Two years later, a recurrence of the above-mentioned carcinoma in multiple locations (the right labial commissure, cheek mucosa, and vestibular fornix) was noted. After extensive discussions between the patient and treating physicians, the decision was made to perform a transoral excision of the tumor with soft-tissue coverage using a contralateral RFFF. The patient provided written informed consent for the surgical procedure. Blood flow through the radial and ulnar systems of the right side was as-

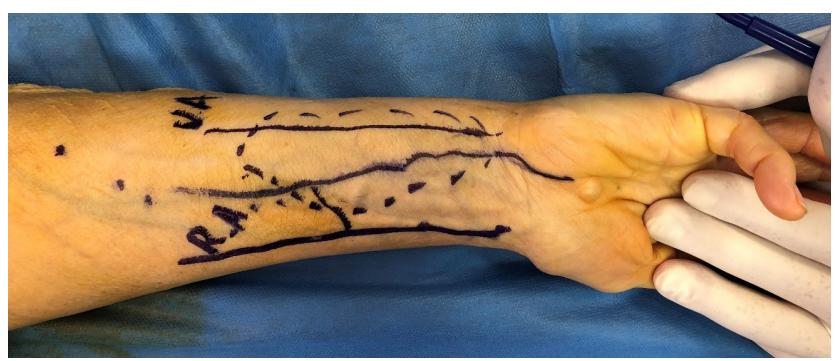

Fig. 1. Preoperative flap markings. The radial artery (RA), ulnar artery (UA), and median artery in between are drawn over the volar forearm surface, according to the ultrasound probe.

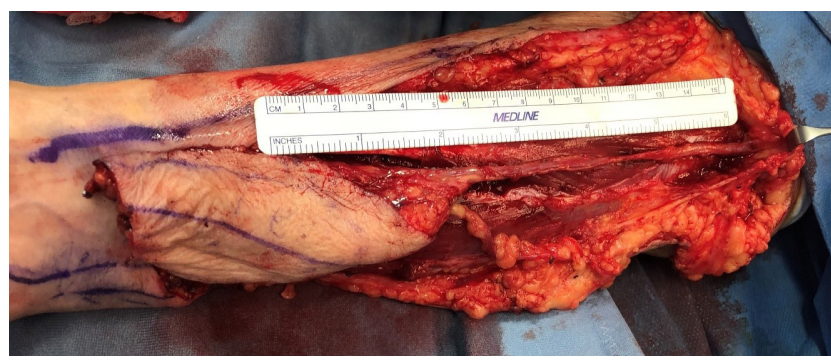

Fig. 2. Median vessel dissection was carried out until the desired pedicle length was achieved $(15 \mathrm{~cm})$. sessed. A handheld US probe revealed the presence of an aberrant median branch of the forearm, while neither radial nor ulnar-sided vascular insufficiencies were identified using the Allen test, which was subsequently performed. The test allowed us to evaluate the safety of sacrificing the median artery. The lesion was excised with wide margins by head and neck cancer surgeons. Frozen sections were obtained to confirm margin clearance. The defect was therefore large, including the right labial commissure and extending up to the right lower alveolar and buccal mucosa. With a template from the defect and according to the US probe, an unusual forearm free flap was marked over the skin of the distal volar forearm (Fig. 1). The plastic surgery team began the elevation of the free flap at the distal margin, first searching for the previously detected median branch. The aberrant artery was seen running between the palmaris longus and flexor carpi radialis tendons, giving off many tiny perforators to the skin paddle at the level of the mid-forearm, and continued deep into the flexor retinaculum toward the palm. The median vessel was strongly pulsatile (Supplemental Video 1), with a caliber of $0.21 \mathrm{~cm}$. Dissection was therefore carried out in a distal-to-proximal fashion up to the division of the artery from the RA (Fig. 2). It was anomalously accompanied by two superficial venae comitantes that proximally joined into a single venous branch (Fig. 3), which was later opportunely anastomosed. As soon as we decided to harvest the flap from the territory of the median artery, we did not dissect the RA along its entire course. Nevertheless, there were no septal branches communicating from the RA to the distal fasciocutaneous skin island, and its vascularity was provided solely by the aberrant median vessel and its perforators. On the contrary, the vascularization of the skin island was supplied by numerous septal perforators arising directly from the median artery (Supplemental Video 2). After applying vascular clamps to the median vessel and

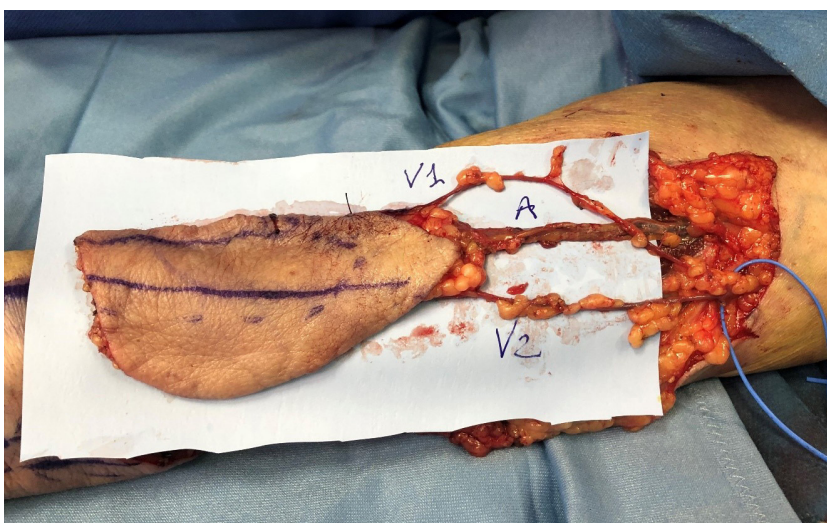

Fig. 3. Complete harvest of the flap. The abnormal forearm free flap was based on the median artery pedicle accompanied by two venae comitantes that proximally joined into a single vein. 


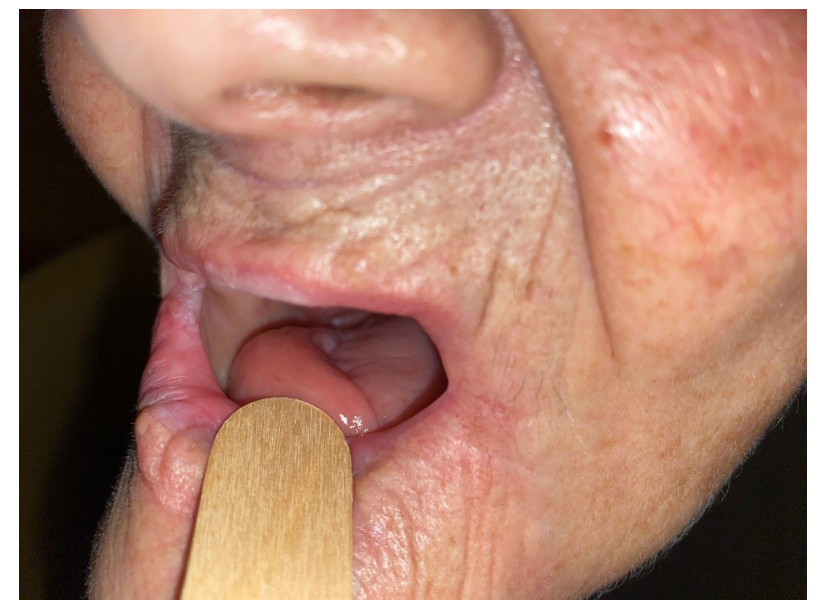

Fig. 4. Six-month postoperative intraoral view. The median forearm flap is successfully inset in the oral cavity.

releasing the tourniquet, a second Allen test was intraoperatively performed. As this maneuver resulted in satisfactory perfusion of the whole hand while compressing the median branch, flap elevation on the anomalous vessel was continued. The flap was inset in the intraoral defect, and the donor site was skin-grafted. No functional deficit was noted in the hand, and the healing of the flap and donor area was normal (Fig. 4).

\section{DISCUSSION}

The fairly consistent structure of the RA system contributes to the success of the RFFF. The RA normally arises from the BA approximately $1 \mathrm{~cm}$ distal to the antecubital fossa, and the usual bifurcation of the BA into the RA and ulnar artery is reported in approximately $70 \%$ of forearms. Nine to 17 septocutaneous (average, 11.7) perforators normally arise from the RA, and most of them are in the distal third of the forearm. At the level of the wrist, the RA usually bifurcates into a superficial palmar branch and a deep palmar branch [12]. The proximal part of the RA is deep to the brachioradialis muscle and cannot be palpated. Distally, it becomes superficial to pronator teres muscle and runs beneath the skin and deep fascia. The RFFF is normally supplied by septocutaneous branches of the RA, which lie between the flexor carpi radialis and brachioradialis muscles. Although variants are rare, many different abnormalities of the arterial system in the forearm have been reported. McCormack et al. [5], in a study of 750 cadaveric extremities, described anatomical variations of the RA in $4.3 \%$ of samples, reporting that the most frequent anomaly discovered was high origin of the RA from the BA. Funk et al. [6], in a series of 52 radial flaps, identified three types of RA anomalies: a superficial dorsal antebrachial artery, a distal take-off of the RA deep to the pronator teres muscle, and a high origin of the RA. Other authors have described high bifurcation of the RA into superficial and deep branches and duplication of the RA [9-11].

A few papers in both the anatomical and reconstructive literature have described a persistent median artery in the forearm. With a prevalence of $5 \%$ to $50 \%$ [13], the frequency of a median artery appeared to have a possible secular trend. More specifically, its presence seemed to be inversely correlated with the date of birth of the individuals evaluated. From the embryological standpoint, the median artery derives from the axial artery and usually regresses at approximately 8 weeks to leave a small accompanying artery of the median nerve. When persistent in adulthood, it has been found to have variable origin and course, with the possibility of arising from any of the major forearm vessels [14]. In most cases, it was accidentally detected while assessing forearm vascularity before RFFF harvesting. To the knowledge of the authors, in the reconstructive literature, the particular variant encountered in this case has never been mentioned and described. In 1994, Niranjan and Shibu [15] reported the opportunistic harvest of a forearm flap based on a perforator of the median artery, which they termed a "median forearm pedicled flap." In 2006, a case of a radial forearm flap based on an intraoperatively detected median vessel was described by Varley et al. [16]. They never encountered its origin, and their report subsequently received a critical commentary [17]. This could be, therefore, the very first forearm free flap based and planned immediately on the median artery, constituting the first anomaly of this type encountered in our practice among more than 100 forearm free flaps. During preoperative planning, the US probe highlighted the presence of a superficial median artery. Upon simultaneous interruption of blood flow through both the ulnar and radial arteries, adequate perfusion of the whole hand was still visible. Therefore, the median artery was believed to actively participate in hand perfusion. Previous cadaveric studies have confirmed that the median artery, when it persists in adult life in the palmar pattern, contributes to the superficial palmar arch [18]. Nonetheless, since hand perfusion was still preserved when blocking the flow through the median vessel, we considered it to be safely expendable.

The thrilling possibility of preserving the major forearm vessels with this free flap prompted us to persevere in our reconstructive plan. During flap elevation, the median artery was found to lie on the surface of the superficial muscular fascia, between the palmaris longus and flexor carpi radialis tendons, accompanied by two superficial venae comitantes that proximally joined into a single venous vessel. We observed that the vascularity of the skin island of the forearm flap was provided solely by the median aberrant vessel and its perforators; therefore, we 
proceeded with the harvest. We dissected the vessels in a distalto-proximal fashion until the desired pedicle length was reached, without encountering the origin of the peculiar vessel. No other vascular abnormalities of the forearm were seen; in particular, the major vessels were normoplastic. The goal of reconstruction was thus successfully achieved, while avoiding the sacrifice of any of the major forearm vessels. Neither hand ischemia nor any donor and recipient site complications were noted.

In conclusion, the rarity of forearm vascular system abnormalities prompted this report in order to better assess the prevalence of persistent median artery and foresee possible risks for forearm flap survival and/or hand ischemia. The preoperative detection of aberrant vessels appears important to prevent damage at the donor site and enable optimal flap harvesting.

\section{NOTES}

\section{Conflict of interest}

Marco Innocenti is an editorial board member of the journal but was not involved in the peer reviewer selection, evaluation, or decision process of this article. No other potential conflicts of interest relevant to this article were reported.

\section{Ethical approval}

All the procedures performed in studies involving human participants were in accordance with the ethical standards of the institutional and national research committee and with the 1964 Helsinki Declaration and its later amendments or comparable ethical standards. Written informed consent was achieved from the patient in this study.

\section{Patient consent}

The patient provided written informed consent for the publication and the use of her images.

\section{Author contribution}

Conceptualization: S Calabrese. Data curation: S Calabrese, N Alfonsi. Formal analysis: S Calabrese, N Alfonsi. Methodology: $S$ Calabrese. Project administration: G Menichini, M Innocenti. Visualization: G Menichini, M Innocenti. Writing - original draft: S Calabrese. Writing - review \& editing: G Menichini, M Innocenti.

\section{ORCID}

Giulio Menichini

Sara Calabrese

Nicola Alfonsi

Marco Innocenti https://orcid.org/0000-0003-1858-3964 https://orcid.org/0000-0001-9066-0704 https://orcid.org/0000-0002-3958-1282 https://orcid.org/0000-0003-3533-1398

\section{Supplementary material}

Supplemental Video 1. After releasing the tourniquet, the median vessel is strongly pulsatile and the median forearm flap appears well vascularized. Supplemental data can be found at: https://doi.org/10.5999/aps.2021.00486.v001

Supplemental Video 2. A focus on the vascularization of this unusual flap. Supplemental data can be found at: https://doi. org/10.5999/aps.2021.00486.v002

\section{REFERENCES}

1. Song R, Gao Y, Song Y, et al. The forearm flap. Clin Plast Surg 1982;9:21-6.

2. Chavre S, Thankappan K, Mathew J, et al. Shape-modified radial forearm free tissue transfer in oral cavity reconstruction: technique and a prospective comparison with the conventional technique. Head Neck 2020;42:3345-51.

3. Tankersley A, Velasco Martinez I, Medina A. Use of cervicothoracic rotation flap and osteocutaneous radial forearm free flap for a complex multilayered cheek defect reconstruction. Case Reports Plast Surg Hand Surg 2020;7:98-104.

4. Jeng SF, Kuo YR, Wei FC, et al. Total lower lip reconstruction with a composite radial forearm-palmaris longus tendon flap: a clinical series. Plast Reconstr Surg 2004;113:1923.

5. McCormack LJ, Cauldwell EW, Anson BJ. Brachial and antebrachial arterial patterns; a study of 750 extremities. Surg Gynecol Obstet 1953;96:43-54.

6. Funk GF, Valentino J, McCulloch TM, et al. Anomalies of forearm vascular anatomy encountered during elevation of the radial forearm flap. Head Neck 1995; 17:284-92.

7. Heden P, Gylbert L. Anomaly of the radial artery encountered during elevation of the radial forearm flap. J Reconstr Microsurg 1990;6:139-41.

8. Sasaki K, Nozaki M, Aiba H, et al. A rare variant of the radial artery: clinical considerations in raising a radial forearm flap. Br J Plast Surg 2000;53:445-7.

9. Bumbasirevic M, Lesic A, Filipovic B. Duplication of the radial artery in the radial forearm flap. Clin Anat 2005; 18:3057.

10. Mordick TG. Vascular variation of the radial forearm flap: a case report.J Reconstr Microsurg 1995; 11:345-6.

11. Acarturk TO, Tuncer U, Aydogan LB, et al. Median artery arising from the radial artery: its significance during harvest of a radial forearm free flap. J Plast Reconstr Aesthet Surg 2008;61:e5-8.

12. Acarturk TO, Newton ED. Aberrant branch of the radial ar- 
tery encountered during elevation of the radial forearm free flap.J Reconstr Microsurg 2004;20:611-4.

13. Henneberg M, George BJ. Possible secular trend in the incidence of an anatomical variant: median artery of the forearm. Am J Phys Anthropol 1995;96:329-34.

14. Rodriguez-Niedenfuhr M, Sanudo JR, Vazquez T, et al. Median artery revisited. J Anat 1999;195(Pt 1):57-63.

15. Niranjan NS, Shibu MM. The median forearm flap. Br J Plast Surg 1994;47:272-4.

16. Varley I, Wales CJ, Carter LM. The median artery: its poten- tial implications for the radial forearm flap. J Plast Reconstr Aesthet Surg 2008;61:693-5.

17. Lloyd MS, Shafighi M, Niranjan N. Response to article, The median artery: its potential complications for the radial forearm flap by Varley et al, JPRAS 61:693-695. J Plast Reconstr Aesthet Surg 2009;62:116.

18. Eid N, Ito Y, Shibata MA, et al. Persistent median artery: cadaveric study and review of the literature. Clin Anat 2011; 24:627-33. 